\title{
Reconstruction of the One-Dimensional Lebesgue Measure
}

\author{
Noboru Endou \\ National Institute of Technology, Gifu College \\ 2236-2 Kamimakuwa, Motosu, Gifu, Japan
}

\begin{abstract}
Summary. In the Mizar system ([1], 2]), Józef Białas has already given the one-dimensional Lebesgue measure 4]. However, the measure introduced by Białas limited the outer measure to a field with finite additivity. So, although it satisfies the nature of the measure, it cannot specify the length of measurable sets and also it cannot determine what kind of set is a measurable set. From the above, the authors first determined the length of the interval by the outer measure. Specifically, we used the compactness of the real space. Next, we constructed the pre-measure by limiting the outer measure to a semialgebra of intervals. Furthermore, by repeating the extension of the previous measure, we reconstructed the one-dimensional Lebesgue measure [7, 3].
\end{abstract}

MSC: 28A12 28A75 68V20

Keywords: Lebesgue measure; algebra of intervals

MML identifier: MEASUR12, version: 8.1.09 5.60.1371

\section{Properties of Intervals}

Now we state the propositions:

(1) Let us consider non empty intervals $A, B$. Suppose $A$ is open interval and $B$ is open interval and $A \cup B$ is an interval. Then

(i) $A \cup B$ is open interval, and

(ii) $A$ meets $B$, and

(iii) $\inf A<\sup B$ or $\inf B<\sup A$. 
(2) Let us consider open interval subsets $A, B$ of $\mathbb{R}$. If $A$ meets $B$, then $A \cup B$ is an open interval subset of $\mathbb{R}$. The theorem is a consequence of (1).

(3) Let us consider an interval $A$, and open interval subsets $B, C$ of $\mathbb{R}$. If $A \subseteq B \cup C$ and $A$ meets $B$ and $A$ meets $C$, then $B$ meets $C$.

Let us consider non empty sets $A, B$ and extended real numbers $p, q, r, s$. Now we state the propositions:

(4) If $A=[p, q]$ and $B=[r, s]$ and $A$ misses $B$, then $q<r$ or $s<p$.

(5) If $A=[p, q]$ and $B=[r, s[$ and $A$ misses $B$, then $q<r$ or $s \leqslant p$.

(6) If $A=[p, q]$ and $B=] r, s]$ and $A$ misses $B$, then $q \leqslant r$ or $s<p$.

(7) If $A=[p, q]$ and $B=] r, s[$ and $A$ misses $B$, then $q \leqslant r$ or $s \leqslant p$.

(8) If $A=[p, q[$ and $B=[r, s[$ and $A$ misses $B$, then $q \leqslant r$ or $s \leqslant p$.

(9) If $A=[p, q[$ and $B=] r, s]$ and $A$ misses $B$, then $q \leqslant r$ or $s<p$.

(10) If $A=[p, q[$ and $B=] r, s[$ and $A$ misses $B$, then $q \leqslant r$ or $s \leqslant p$.

(11) If $A=] p, q]$ and $B=] r, s]$ and $A$ misses $B$, then $q \leqslant r$ or $s \leqslant p$.

(12) If $A=] p, q]$ and $B=] r, s[$ and $A$ misses $B$, then $q \leqslant r$ or $s \leqslant p$.

(13) If $A=] p, q[$ and $B=] r, s[$ and $A$ misses $B$, then $q \leqslant r$ or $s \leqslant p$.

(14) Let us consider non empty intervals $A, B$, and extended real numbers $p$, $q, r, s$. Suppose $A=[p, q]$ and $B=[r, s]$ and $A$ misses $B$. Then $A \cup B$ is not an interval. The theorem is a consequence of (4).

Let us consider non empty intervals $A, B$ and extended real numbers $p, q$, $r, s$. Now we state the propositions:

(15) If $A=[p, q]$ and $B=[r, s[$ and $A$ misses $B$ and $A \cup B$ is an interval, then $p=s$ and $A \cup B=[r, q]$. The theorem is a consequence of (5).

(16) If $A=[p, q]$ and $B=] r, s]$ and $A$ misses $B$ and $A \cup B$ is an interval, then $q=r$ and $A \cup B=[p, s]$. The theorem is a consequence of (6).

(17) Suppose $A=[p, q]$ and $B=] r, s[$ and $A$ misses $B$ and $A \cup B$ is an interval. Then

(i) $p=s$ and $A \cup B=] r, q]$, or

(ii) $q=r$ and $A \cup B=[p, s[$.

The theorem is a consequence of (7).

(18) Suppose $A=[p, q[$ and $B=[r, s[$ and $A$ misses $B$ and $A \cup B$ is an interval. Then

(i) $p=s$ and $A \cup B=[r, q[$, or

(ii) $q=r$ and $A \cup B=[p, s[$.

The theorem is a consequence of (8). 
(19) Let us consider non empty intervals $A, B$, and extended real numbers $p$, $q, r, s$. Suppose $A=[p, q[$ and $B=] r, s]$ and $A$ misses $B$. Then $A \cup B$ is not an interval. The theorem is a consequence of (9).

Let us consider non empty intervals $A, B$ and extended real numbers $p, q$, $r, s$. Now we state the propositions:

(20) Suppose $A=[p, q[$ and $B=] r, s[$ and $A$ misses $B$ and $A \cup B$ is an interval. Then

(i) $p=s$, and

(ii) $A \cup B=] r, q[$.

The theorem is a consequence of (10).

(21) Suppose $A=] p, q]$ and $B=] r, s]$ and $A$ misses $B$ and $A \cup B$ is an interval. Then

(i) $p=s$ and $A \cup B=] r, q]$, or

(ii) $q=r$ and $A \cup B=] p, s]$.

The theorem is a consequence of (11).

(22) Suppose $A=] p, q]$ and $B=] r, s[$ and $A$ misses $B$ and $A \cup B$ is an interval. Then

(i) $q=r$, and

(ii) $A \cup B=] p, s[$.

The theorem is a consequence of (12).

(23) Let us consider non empty intervals $A, B$, and extended real numbers $p$, $q, r, s$. Suppose $A=] p, q[$ and $B=] r, s[$ and $A$ misses $B$. Then $A \cup B$ is not an interval. The theorem is a consequence of (13).

(24) Let us consider real numbers $a, b$, and a subset $I$ of $\mathbb{R}^{\mathbf{1}}$. If $I=[a, b]$, then $I$ is compact.

\section{Tools for Extended Real Sequences}

Let $f$ be a finite sequence of elements of $\overline{\mathbb{R}}$. The functor $\max _{\mathrm{p}} f$ yielding a natural number is defined by

(Def. 1) if len $f=0$, then it $=0$ and if len $f>0$, then $i t \in \operatorname{dom} f$ and for every natural number $i$ and for every extended reals $r_{1}, r_{2}$ such that $i \in \operatorname{dom} f$ and $r_{1}=f(i)$ and $r_{2}=f(i t)$ holds $r_{1} \leqslant r_{2}$ and for every natural number $j$ such that $j \in \operatorname{dom} f$ and $f(j)=f(i t)$ holds $i t \leqslant j$.

The functor $\min _{\mathrm{p}} f$ yielding a natural number is defined by 
(Def. 2) if len $f=0$, then $i t=0$ and if $\operatorname{len} f>0$, then $i t \in \operatorname{dom} f$ and for every natural number $i$ and for every extended reals $r_{1}, r_{2}$ such that $i \in \operatorname{dom} f$ and $r_{1}=f(i)$ and $r_{2}=f(i t)$ holds $r_{1} \geqslant r_{2}$ and for every natural number $j$ such that $j \in \operatorname{dom} f$ and $f(j)=f(i t)$ holds $i t \leqslant j$.

The functors: $\max f$ and $\min f$ yielding extended reals are defined by terms

(Def. 3) $f\left(\max _{\mathrm{p}} f\right)$,

(Def. 4) $f\left(\min _{\mathrm{p}} f\right)$,

respectively.

Let us consider a finite sequence $f$ of elements of $\overline{\mathbb{R}}$ and a natural number $i$. Now we state the propositions:

(25) If $1 \leqslant i \leqslant \operatorname{len} f$, then $f(i) \leqslant f\left(\max _{\mathrm{p}} f\right)$ and $f(i) \leqslant \max f$.

(26) If $1 \leqslant i \leqslant \operatorname{len} f$, then $f(i) \geqslant f\left(\min _{\mathrm{p}} f\right)$ and $f(i) \geqslant \min f$.

Let us consider a function $F$ and objects $x, y$. Now we state the propositions:

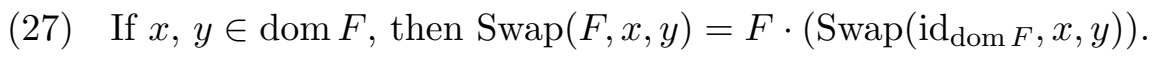

(28) If $x, y \in \operatorname{dom} F$, then $F$ and $\operatorname{Swap}(F, x, y)$ are fiberwise equipotent. The theorem is a consequence of (27).

Now we state the proposition:

(29) Let us consider a set $X$, a function $F$, and objects $x, y$. Suppose $x \notin X$ and $y \notin X$. Then $F\lceil X=\operatorname{Swap}(F, x, y) \uparrow X$.

\section{Open Covering of Intervals}

Let $A$ be a subset of $\mathbb{R}$.

An open interval covering of $A$ is an interval covering of $A$ defined by

(Def. 5) for every element $n$ of $\mathbb{N}, i t(n)$ is open interval.

Let $F$ be an open interval covering of $A$ and $n$ be an element of $\mathbb{N}$. One can verify that the functor $F(n)$ yields an open interval subset of $\mathbb{R}$. Let $F$ be a sequence of $2^{\mathbb{R}}$.

An open interval covering of $F$ is an interval covering of $F$ defined by

(Def. 6) for every element $n$ of $\mathbb{N}$, it $(n)$ is an open interval covering of $F(n)$.

Let $H$ be an open interval covering of $F$ and $n$ be an element of $\mathbb{N}$. Let us note that the functor $H(n)$ yields an open interval covering of $F(n)$. Let $A$ be a subset of $\mathbb{R}$. The functor $\operatorname{Svc} 2(A)$ yielding a subset of $\overline{\mathbb{R}}$ is defined by

(Def. 7) for every extended real number $x, x \in$ it iff there exists an open interval covering $F$ of $A$ such that $x=\operatorname{vol}(F)$.

Let us note that $\operatorname{Svc} 2(A)$ is non empty. Now we state the propositions:

(30) Let us consider a subset $A$ of $\mathbb{R}$. Then 
(i) $\operatorname{Svc} 2(A) \subseteq \operatorname{Svc}(A)$, and

(ii) $\inf \operatorname{Svc}(A) \leqslant \inf \operatorname{Svc} 2(A)$.

(31) Let us consider a sequence $F$ of $2^{\mathbb{R}}$, an open interval covering $G$ of $F$, and a sequence $H$ of $\mathbb{N} \times \mathbb{N}$. Suppose $\operatorname{rng} H=\mathbb{N} \times \mathbb{N}$. Then $\operatorname{On}(G, H)$ is an open interval covering of $\bigcup \operatorname{rng} F$.

(32) Let us consider a subset $A$ of $\mathbb{R}$, and a sequence $G$ of $2^{\mathbb{R}}$. Suppose $A \subseteq$ $\cup \operatorname{rng} G$ and for every element $n$ of $\mathbb{N}, G(n)$ is open interval. Then $G$ is an open interval covering of $A$.

(33) Let us consider a sequence $F$ of $2^{\mathbb{R}}$, and a sequence $G$ of $\left(2^{\mathbb{R}}\right)^{\mathbb{N}}$. Suppose for every element $n$ of $\mathbb{N}, G(n)$ is an open interval covering of $F(n)$. Then $G$ is an open interval covering of $F$.

(34) Let us consider a sequence $H$ of $\mathbb{N} \times \mathbb{N}$. Suppose $H$ is one-to-one and $\operatorname{rng} H=\mathbb{N} \times \mathbb{N}$. Let us consider a natural number $k$. Then there exists an element $m$ of $\mathbb{N}$ such that for every sequence $F$ of $2^{\mathbb{R}}$ for every open interval covering $G$ of $F$, $(\operatorname{Ser}((\operatorname{On}(G, H)) \operatorname{vol}))(k) \leqslant(\operatorname{Ser} \operatorname{vol}(G))(m)$.

(35) Let us consider a sequence $F$ of $2^{\mathbb{R}}$, and an open interval covering $G$ of $F$. Then $\inf \operatorname{Svc} 2(\bigcup \operatorname{rng} F) \leqslant \sum \operatorname{vol}(G)$. The theorem is a consequence of (34) and (31).

Let $F$ be a non empty family of subsets of $\mathbb{R}$. One can verify that an element of $F$ is a subset of $\mathbb{R}$. Now we state the propositions:

(36) Let us consider an element $A$ of Intervals $\mathbb{R}_{\mathbb{R}}$. Suppose $A$ is open interval. Then there exists an open interval covering $F$ of $A$ such that

(i) $F(0)=A$, and

(ii) for every natural number $n$ such that $n \neq 0$ holds $F(n)=\emptyset$, and

(iii) $\bigcup \operatorname{rng} F=A$, and

(iv) $\bar{\sum}((F) \mathrm{vol})=\varnothing A$.

Proof: Define $\mathcal{P}$ [natural number, set] $\equiv$ if $\$_{1}=0$, then $\$_{2}=A$ and if $\$_{1} \neq 0$, then $\$_{2}=\emptyset_{\mathbb{R}}$. For every element $n$ of $\mathbb{N}$, there exists an element $E$ of $2^{\mathbb{R}}$ such that $\mathcal{P}[n, E]$. Consider $F$ being a function from $\mathbb{N}$ into $2^{\mathbb{R}}$ such that for every element $n$ of $\mathbb{N}, \mathcal{P}[n, F(n)]$. For every natural number $n$ such that $n \neq 0$ holds $F(n)=\emptyset$. For every object $n, 0 \leqslant((F) \operatorname{vol})(n)$. Define $\mathcal{P}$ [natural number] $\equiv\left(\left(\sum_{\alpha=0}^{\kappa}(F) \operatorname{vol}(\alpha)\right)_{\kappa \in \mathbb{N}}\right)\left(\$_{1}\right)=\varnothing A$. For every natural number $n$ such that $\mathcal{P}[n]$ holds $\mathcal{P}[n+1]$. For every natural number $n, \mathcal{P}[n] . \bar{\sum}((F) \operatorname{vol})=\varnothing A$ by [6, (2)], [8, (32)], [5, (52)].

(37) Let us consider subsets $A, B$ of $\mathbb{R}$, and an interval covering $F$ of $A$. If $B \subseteq A$, then $F$ is an interval covering of $B$. 
(38) Let us consider subsets $A, B$ of $\mathbb{R}$, and an open interval covering $F$ of $A$. If $B \subseteq A$, then $F$ is an open interval covering of $B$. The theorem is a consequence of (37).

(39) Let us consider subsets $A, B$ of $\mathbb{R}$, an interval covering $F$ of $A$, and an interval covering $G$ of $B$. If $F=G$, then $(F)$ vol $=(G)$ vol.

(40) Let us consider a finite sequence $F$ of elements of $2^{\mathbb{R}}$, and a natural number $k$. Suppose for every natural number $n$ such that $n \in \operatorname{dom} F$ holds $F(n)$ is an open interval subset of $\mathbb{R}$ and for every natural number $n$ such that $1 \leqslant n<$ len $F$ holds $\bigcup \operatorname{rng}(F\lceil n)$ meets $F(n+1)$. Then $\bigcup \operatorname{rng}(F\lceil k)$ is an open interval subset of $\mathbb{R}$.

Proof: Define $\mathcal{P}$ [natural number $] \equiv \bigcup \operatorname{rng}\left(F\left\lceil \$_{1}\right)\right.$ is an open interval subset of $\mathbb{R}$. For every natural number $k$ such that $\mathcal{P}[k]$ holds $\mathcal{P}[k+1]$. For every natural number $k, \mathcal{P}[k]$.

(41) Let us consider a non empty, closed interval subset $A$ of $\mathbb{R}$, and a finite sequence $F$ of elements of $2^{\mathbb{R}}$. Suppose $A \subseteq \bigcup \operatorname{rng} F$ and for every natural number $n$ such that $n \in \operatorname{dom} F$ holds $A$ meets $F(n)$ and for every natural number $n$ such that $n \in \operatorname{dom} F$ holds $F(n)$ is an open interval subset of $\mathbb{R}$. Then there exists a finite sequence $G$ of elements of $2^{\mathbb{R}}$ such that

(i) $F$ and $G$ are fiberwise equipotent, and

(ii) for every natural number $n$ such that $1 \leqslant n<\operatorname{len} G$ holds $\bigcup \operatorname{rng}(G\lceil n)$ meets $G(n+1)$.

Proof: Define $\mathcal{P}$ [natural number] $\equiv$ if $\$_{1} \leqslant$ len $F$, then there exists a finite sequence $G$ of elements of $2^{\mathbb{R}}$ such that $F$ and $G$ are fiberwise equipotent and for every natural number $n$ such that $1 \leqslant n<\$_{1}$ holds $\bigcup \operatorname{rng}(G\lceil n)$ meets $G(n+1)$. For every non zero natural number $k$ such that $\mathcal{P}[k]$ holds $\mathcal{P}[k+1]$. For every non zero natural number $k, \mathcal{P}[k]$. Consider $G$ being a finite sequence of elements of $2^{\mathbb{R}}$ such that $F$ and $G$ are fiberwise equipotent and for every natural number $n$ such that $1 \leqslant n<\operatorname{len} F$ holds $\bigcup \operatorname{rng}(G\lceil n)$ meets $G(n+1)$.

\section{Measure of Intervals by OS-Meas}

Let us consider an element $I$ of Intervals $s_{\mathbb{R}}$. Now we state the propositions:

(42) If $I$ is open interval, then $($ OS-Meas $)(I) \leqslant \varnothing I$. The theorem is a consequence of (36) and (30).

(43) If $I \neq \emptyset$ and $I$ is right open interval, then (OS-Meas) $(I) \leqslant \varnothing I$. The theorem is a consequence of $(36),(38),(39)$, and (30). 
(44) If $I$ is an interval, then $(\mathrm{OS}-\mathrm{Meas})(I) \leqslant \varnothing I$. The theorem is a consequence of (42) and (43).

(45) Let us consider a non empty, closed interval subset $A$ of $\mathbb{R}$, a finite sequence $F$ of elements of $2^{\mathbb{R}}$, and a finite sequence $G$ of elements of $\overline{\mathbb{R}}$. Suppose $A \subseteq \bigcup \operatorname{rng} F$ and len $F=\operatorname{len} G$ and for every natural number $n$ such that $n \in \operatorname{dom} F$ holds $F(n)$ is an open interval subset of $\mathbb{R}$ and for every natural number $n$ such that $n \in \operatorname{dom} F$ holds $G(n)=\varnothing F(n)$ and for every natural number $n$ such that $n \in \operatorname{dom} F$ holds $A$ meets $F(n)$. Then $\varnothing A \leqslant \sum G$.

Proof: Consider $F_{1}$ being a finite sequence of elements of $2^{\mathbb{R}}$ such that $F$ and $F_{1}$ are fiberwise equipotent and for every natural number $n$ such that $1 \leqslant n<\operatorname{len} F_{1}$ holds $\bigcup \operatorname{rng}\left(F_{1}\lceil n)\right.$ meets $F_{1}(n+1)$. Consider $P$ being a permutation of $\operatorname{dom} F$ such that $F=F_{1} \cdot P$. Reconsider $G_{1}=G \cdot\left(P^{-1}\right)$ as a finite sequence of elements of $\overline{\mathbb{R}}$. For every natural number $n$ such that $n \in \operatorname{dom} F_{1}$ holds $G_{1}(n)=\varnothing F_{1}(n)$. Define $\mathcal{P}$ [natural number] $\equiv$ if $\$_{1} \in \operatorname{dom} F_{1}$, then $\varnothing \bigcup \operatorname{rng}\left(F_{1} \uparrow \$_{1}\right) \leqslant \sum\left(G_{1} \uparrow \$_{1}\right)$. For every natural number $k$ such that $\mathcal{P}[k]$ holds $\mathcal{P}[k+1]$. For every natural number $k, \mathcal{P}[k]$. $\bigcup \operatorname{rng}\left(F_{1} \uparrow \operatorname{len} F_{1}\right)$ is an open interval subset of $\mathbb{R}$.

(46) Let us consider a non empty set $X$, a sequence $f$ of $X$, and natural numbers $i, j$. Then there exists a sequence $g$ of $X$ such that

(i) for every natural number $n$ such that $n \neq i$ and $n \neq j$ holds $f(n)=$ $g(n)$, and

(ii) $f(i)=g(j)$, and

(iii) $f(j)=g(i)$.

Proof: Define $\mathcal{P}$ [object, object] $\equiv$ if $\$_{1} \neq i$ and $\$_{1} \neq j$, then $\$_{2}=f\left(\$_{1}\right)$ and if $\$_{1}=i$, then $\$_{2}=f(j)$ and if $\$_{1}=j$, then $\$_{2}=f(i)$. For every element $n$ of $\mathbb{N}$, there exists an element $x$ of $X$ such that $\mathcal{P}[n, x]$. Consider $g$ being a function from $\mathbb{N}$ into $X$ such that for every element $n$ of $\mathbb{N}$, $\mathcal{P}[n, g(n)]$.

(47) Let us consider sequences $f, g$ of $\overline{\mathbb{R}}$. Suppose $f$ is non-negative and there exists a natural number $N$ such that $(\operatorname{Ser} f)(N) \leqslant(\operatorname{Ser} g)(N)$ and for every natural number $n$ such that $n>N$ holds $f(n) \leqslant g(n)$. Then $\bar{\sum} f \leqslant \bar{\sum} g$. Proof: Consider $N$ being a natural number such that $(\operatorname{Ser} f)(N) \leqslant$ $(\operatorname{Ser} g)(N)$ and for every natural number $n$ such that $n>N$ holds $f(n) \leqslant$ $g(n)$. Define $\mathcal{P}$ [natural number] $\equiv(\operatorname{Ser} f)\left(N+\$_{1}\right) \leqslant(\operatorname{Ser} g)\left(N+\$_{1}\right)$. For every natural number $k$ such that $\mathcal{P}[k]$ holds $\mathcal{P}[k+1]$. For every natural number $m, \mathcal{P}[m]$. For every extended real $x$ such that $x \in \operatorname{rng}$ Ser $f$ there exists an extended real $y$ such that $y \in \operatorname{rng} \operatorname{Ser} g$ and $x \leqslant y$. 
(48) Let us consider sequences $f, g$ of $\overline{\mathbb{R}}$, and natural numbers $j, k$. Suppose $k<j$ and for every natural number $n$ such that $n<j$ holds $f(n)=g(n)$. Then $(\operatorname{Ser} f)(k)=(\operatorname{Ser} g)(k)$.

Proof: Define $\mathcal{P}$ [natural number] $\equiv$ if $\$_{1} \leqslant k$, then $($ Ser $f)\left(\$_{1}\right)=$ $(\operatorname{Ser} g)\left(\$_{1}\right)$. For every natural number $m$ such that $\mathcal{P}[m]$ holds $\mathcal{P}[m+1]$. For every natural number $m, \mathcal{P}[m]$.

(49) Let us consider sequences $f, g$ of $\overline{\mathbb{R}}$, and natural numbers $i, j$. Suppose $f$ is non-negative and $i \geqslant j$ and for every natural number $n$ such that $n \neq i$ and $n \neq j$ holds $f(n)=g(n)$ and $f(i)=g(j)$ and $f(j)=g(i)$. Then $(\operatorname{Ser} f)(i)=(\operatorname{Ser} g)(i)$.

Proof: For every element $k$ of $\mathbb{N}, 0 \leqslant g(k)$.

(50) Let us consider sequences $f, g$ of $\overline{\mathbb{R}}$, and natural numbers $i, j$. Suppose $f$ is non-negative and $f(i)=g(j)$ and $f(j)=g(i)$ and for every natural number $n$ such that $n \neq i$ and $n \neq j$ holds $f(n)=g(n)$. Let us consider a natural number $n$. If $n \geqslant i$ and $n \geqslant j$, then $(\operatorname{Ser} f)(n)=(\operatorname{Ser} g)(n)$.

Proof: Define $\mathcal{P}$ [natural number] $\equiv$ if $\$_{1} \geqslant i$ and $\$_{1} \geqslant j$, then $($ Ser $f)\left(\$_{1}\right)=$ $(\operatorname{Ser} g)\left(\$_{1}\right)$. For every natural number $k$ such that $\mathcal{P}[k]$ holds $\mathcal{P}[k+1]$. For every natural number $k, \mathcal{P}[k]$.

(51) Let us consider sequences $f, g$ of $\overline{\mathbb{R}}$, and natural numbers $i, j$. Suppose $f$ is non-negative and $i \geqslant j$ and for every natural number $n$ such that $n \neq i$ and $n \neq j$ holds $f(n)=g(n)$ and $f(i)=g(j)$ and $f(j)=g(i)$. Then $\bar{\sum} f=\bar{\sum} g$.

Proof: For every element $k$ of $\mathbb{N}, 0 \leqslant g(k)$.

(52) Let us consider a subset $A$ of $\mathbb{R}$, interval coverings $F_{1}, F_{2}$ of $A$, and natural numbers $n, m$. Suppose for every natural number $k$ such that $k \neq n$ and $k \neq m$ holds $F_{1}(k)=F_{2}(k)$ and $F_{1}(n)=F_{2}(m)$ and $F_{1}(m)=F_{2}(n)$. Then $\operatorname{vol}\left(F_{1}\right)=\operatorname{vol}\left(F_{2}\right)$. The theorem is a consequence of $(51)$.

(53) Let us consider a subset $A$ of $\mathbb{R}$, interval coverings $F_{1}, F_{2}$ of $A$, and natural numbers $n, m$. Suppose for every natural number $k$ such that $k \neq n$ and $k \neq m$ holds $F_{1}(k)=F_{2}(k)$ and $F_{1}(n)=F_{2}(m)$ and $F_{1}(m)=F_{2}(n)$. Let us consider a natural number $k$. Suppose $k \geqslant n$ and $k \geqslant m$. Then $\left(\operatorname{Ser}\left(\left(F_{1}\right) \operatorname{vol}\right)\right)(k)=\left(\operatorname{Ser}\left(\left(F_{2}\right) \operatorname{vol}\right)\right)(k)$. The theorem is a consequence of (50).

(54) Let us consider a non empty set $X$, a sequence $s_{2}$ of $X$, and a finite sequence $f$ of elements of $X$. Suppose $\operatorname{rng} f \subseteq \operatorname{rng} s_{2}$. Then there exists a natural number $N$ such that $\operatorname{rng} f \subseteq \operatorname{rng}\left(s_{2} \mid \mathbb{Z}_{N}\right)$.

Proof: Define $\mathcal{P}$ [natural number] $\equiv$ for every finite sequence $F$ of elements of $X$ such that len $F=\$_{1}$ and $\operatorname{rng} F \subseteq \operatorname{rng} s_{2}$ there exists a natural number $N$ such that $\operatorname{rng} F \subseteq \operatorname{rng}\left(s_{2} \mid \mathbb{Z}_{N}\right)$. For every natural number $k$ 
such that $\mathcal{P}[k]$ holds $\mathcal{P}[k+1]$. For every natural number $k, \mathcal{P}[k]$.

(55) Let us consider a non empty subset $A$ of $\mathbb{R}$, an interval covering $F$ of $A$, and a one-to-one finite sequence $G$ of elements of $2^{\mathbb{R}}$. Suppose $\operatorname{rng} G \subseteq$ rng $F$. Then there exists an interval covering $F_{1}$ of $A$ such that

(i) for every natural number $n$ such that $n \in \operatorname{dom} G$ holds $G(n)=F_{1}(n)$, and

(ii) $\operatorname{vol}\left(F_{1}\right)=\operatorname{vol}(F)$.

Proof: Define $\mathcal{P}$ [natural number] $\equiv$ there exists an interval covering $F_{0}$ of $A$ such that for every natural number $n$ such that $n \in \operatorname{dom}\left(G \uparrow \$_{1}\right)$ holds $\left(G \uparrow \$_{1}\right)(n)=F_{0}(n)$ and $F_{0}$ and $F$ are fiberwise equipotent and $\operatorname{vol}\left(F_{0}\right)=$ $\operatorname{vol}(F) . \mathcal{P}[0]$. For every natural number $k$ such that $\mathcal{P}[k]$ holds $\mathcal{P}[k+1]$. For every natural number $k, \mathcal{P}[k]$.

(56) Let us consider a non empty subset $A$ of $\mathbb{R}$, an interval covering $F$ of $A$, a one-to-one finite sequence $G$ of elements of $2^{\mathbb{R}}$, and a finite sequence $H$ of elements of $\overline{\mathbb{R}}$. Suppose $\operatorname{rng} G \subseteq \operatorname{rng} F$ and $\operatorname{dom} G=\operatorname{dom} H$ and for every natural number $n, H(n)=\varnothing G(n)$. Then $\sum H \leqslant \operatorname{vol}(F)$.

Proof: Consider $F_{1}$ being an interval covering of $A$ such that for every natural number $n$ such that $n \in \operatorname{dom} G$ holds $G(n)=F_{1}(n)$ and $\operatorname{vol}\left(F_{1}\right)=$ $\operatorname{vol}(F)$. Consider $S$ being a sequence of $\overline{\mathbb{R}}$ such that $\sum H=S($ len $H)$ and $S(0)=0$ and for every natural number $n$ such that $n<$ len $H$ holds $S(n+1)=S(n)+H(n+1)$. Define $\mathcal{P}$ [natural number] $\equiv$ if $\$_{1} \leqslant$ len $H$, then $S\left(\$_{1}\right) \leqslant\left(\operatorname{Ser}\left(\left(F_{1}\right)\right.\right.$ vol $\left.)\right)\left(\$_{1}\right)$. For every natural number $n$ such that $\mathcal{P}[n]$ holds $\mathcal{P}[n+1]$. For every natural number $n, \mathcal{P}[n]$.

(57) Let us consider an interval $I$. Then $\varnothing I=($ OS-Meas $)(I)$. The theorem is a consequence of (44).

\section{Construction of the One-Dimensional Lebesgue Measure}

Let $F$ be a finite sequence of elements of Intervals $_{\mathbb{R}}$ and $n$ be a natural number. Let us note that the functor $F(n)$ yields an interval subset of $\mathbb{R}$. The functor pre-Meas yielding a non-negative, zeroed function from Intervals $\mathbb{R}$ into $\overline{\mathbb{R}}$ is defined by the term

(Def. 8) OS-Meas $\uparrow$ Intervals $_{\mathbb{R}}$.

Now we state the propositions:

(58) Let us consider an element $I$ of Intervals $\mathbb{R}_{\mathbb{R}}$. Then (pre-Meas) $(I)=\varnothing I$. The theorem is a consequence of (57).

(59) Let us consider an interval $I$. Then (pre-Meas) $(I)=\varnothing I$. The theorem is a consequence of (58). 
(60) Let us consider elements $A, B$ of Intervals $\mathbb{R}_{\mathbb{R}}$. Suppose $A$ misses $B$ and $A \cup$ $B$ is an interval. Then (pre-Meas $)(A \cup B)=($ pre-Meas $)(A)+($ pre-Meas $)(B)$. The theorem is a consequence of (58), (14), (15), (59), (16), (17), (19), (18), (20), (21), (22), and (23).

(61) Let us consider a non empty, disjoint valued finite sequence $F$ of elements of Intervals $\mathbb{R}_{\mathbb{R}}$. Suppose $\cup F$ is an interval. Then there exists a natural number $n$ such that

(i) $n \in \operatorname{dom} F$, and

(ii) $(\bigcup F) \backslash F(n)$ is an interval.

The theorem is a consequence of (26).

(62) Let us consider an interval $A$. Then (pre-Meas) $\cdot\langle A\rangle=\langle($ pre-Meas $)(A)\rangle$. Proof: Reconsider $F=\langle A\rangle$ as a finite sequence of elements of Intervals $\mathbb{R}_{\mathbb{R}}$. For every natural number $n$ such that $n \in \operatorname{dom}(($ pre-Meas $) \cdot F)$ holds $(($ pre-Meas $) \cdot F)(n)=\langle($ pre-Meas $)(A)\rangle(n)$.

(63) Let us consider a disjoint valued finite sequence $F$ of elements of Intervals $\mathbb{R}_{\mathbb{R}}$. Suppose $\bigcup F \in$ Intervals $_{\mathbb{R}}$. Then there exists a disjoint valued finite sequence $G$ of elements of Intervals $\mathbb{R}_{\mathbb{R}}$ such that

(i) $F$ and $G$ are fiberwise equipotent, and

(ii) for every natural number $n$ such that $n \in \operatorname{dom} G$ holds $\bigcup(G\lceil n) \in$ Intervals $_{\mathbb{R}}$ and (pre-Meas) $\left(\bigcup(G\lceil n))=\sum\right.$ (pre-Meas) $\cdot(G \nmid n)$.

Proof: Define $\mathcal{P}$ [natural number] $\equiv$ for every disjoint valued finite sequence $H$ of elements of Intervals $\mathbb{R}_{\mathbb{R}}$ such that len $H=\$_{1}$ and $\bigcup H \in$ Intervals $\mathbb{R}_{\mathbb{R}}$ there exists a disjoint valued finite sequence $G$ of elements of Intervals $_{\mathbb{R}}$ such that $H$ and $G$ are fiberwise equipotent and for every natural number $n$ such that $n \in \operatorname{dom} G$ holds $\bigcup\left(G\lceil n) \in\right.$ Intervals $_{\mathbb{R}}$ and (pre-Meas) $(\bigcup(G \nmid n))=\sum$ (pre-Meas) $\cdot(G\lceil n)$. For every natural number $k$ such that $\mathcal{P}[k]$ holds $\mathcal{P}[k+1]$. For every natural number $k, \mathcal{P}[k]$.

(64) Let us consider finite sequences $F, G$ of elements of $\overline{\mathbb{R}}$. Then

(i) if $F$ is without $-\infty$ and $G$ is without $-\infty$, then $F \frown G$ is without $-\infty$, and

(ii) if $F$ is without $+\infty$ and $G$ is without $+\infty$, then $F^{\frown} G$ is without $+\infty$.

(65) Let us consider a finite sequence $F$ of elements of $\overline{\mathbb{R}}$, and a natural number $k$. Then

(i) if $F$ is without $-\infty$, then $F_{\downarrow k}$ is without $-\infty$, and

(ii) if $F$ is without $+\infty$, then $F_{l k}$ is without $+\infty$. 
(66) Let us consider a finite sequence $F$ of elements of $\overline{\mathbb{R}}$. Then

(i) if $F$ is without $-\infty$, then $\sum F \neq-\infty$, and

(ii) if $F$ is without $+\infty$, then $\sum F \neq+\infty$.

Proof: Consider $S$ being a sequence of $\overline{\mathbb{R}}$ such that $\sum F=S($ len $F)$ and $S(0)=0$ and for every natural number $n$ such that $n<\operatorname{len} F$ holds $S(n+1)=S(n)+F(n+1)$. Define $\mathcal{P}$ [natural number] $\equiv$ if $\$_{1} \leqslant$ len $F$, then $S\left(\$_{1}\right) \neq+\infty$. For every natural number $n$ such that $\mathcal{P}[n]$ holds $\mathcal{P}[n+1]$. For every natural number $n, \mathcal{P}[n]$.

(67) Let us consider without $-\infty$ finite sequences $R_{1}, R_{2}$ of elements of $\overline{\mathbb{R}}$. If $R_{1}$ and $R_{2}$ are fiberwise equipotent, then $\sum R_{1}=\sum R_{2}$.

Proof: Define $\mathcal{P}$ [natural number] $\equiv$ for every without $-\infty$ finite sequences $f, g$ of elements of $\overline{\mathbb{R}}$ such that $f$ and $g$ are fiberwise equipotent and len $f=\$_{1}$ holds $\sum f=\sum g$. For every natural number $n$ such that $\mathcal{P}[n]$ holds $\mathcal{P}[n+1] . \mathcal{P}[0]$. For every natural number $n, \mathcal{P}[n]$.

(68) Let us consider a disjoint valued finite sequence $F$ of elements of Intervals $\mathrm{s}_{\mathbb{R}}$. Suppose $\bigcup F \in$ Intervals $_{\mathbb{R}}$. Then (pre-Meas) $(\bigcup F)=\sum$ (pre-Meas) $F$. The theorem is a consequence of (63), (59), and (67).

(69) Let us consider a disjoint valued function $K$ from $\mathbb{N}$ into Intervals $\mathbb{R}_{\mathbb{R}}$. Suppose $\bigcup K \in$ Intervals $_{\mathbb{R}}$. Then (pre-Meas) $(\bigcup K) \leqslant \bar{\sum}$ (pre-Meas) $\cdot K$.

Proof: Reconsider $F=K$ as a sequence of $2^{\mathbb{R}}$. For every element $n$ of $\mathbb{N}$, $(($ OS-Meas $) \cdot F)(n)=(($ pre-Meas $) \cdot K)(n)$.

One can verify that the functor pre-Meas yields a pre-measure of Intervals $\mathbb{R}_{\mathbb{R}}$. The functor J-Meas yielding a measure on the field generated by Intervals $\mathbb{R}_{\mathbb{R}}$ is defined by

(Def. 9) for every set $A$ such that $A \in$ the field generated by Intervals $\mathbb{R}$ for every disjoint valued finite sequence $F$ of elements of Intervals $\mathbb{R}_{\mathbb{R}}$ such that $A=\bigcup F$ holds it $(A)=\sum($ pre-Meas $) \cdot F$.

Note that the functor J-Meas yields an induced measure of Intervals $\mathbb{R}$ and pre-Meas. Now we state the proposition:

(70) J-Meas is completely-additive.

The functor B-Meas yielding a $\sigma$-measure on the Borel sets is defined by the term

(Def. 10) $\sigma$-Meas(the Caratheodory measure determined by J-Meas) $\uparrow($ the Borel sets).

Let us consider an interval $A$. Now we state the propositions:

(71) $(\mathrm{J}-$ Meas $)(A)=\varnothing A$. The theorem is a consequence of (62) and (59).

(72) $($ B-Meas $)(A)=\varnothing A$. The theorem is a consequence of $(71)$. 
(73) $A \in$ the Borel sets.

The functor L-Field yielding a $\sigma$-field of subsets of $\mathbb{R}$ is defined by the term (Def. 11) COM(the Borel sets, B-Meas).

The functor L-Meas yielding a $\sigma$-measure on L-Field is defined by the term (Def. 12) COM(B-Meas).

Observe that L-Meas is complete. Now we state the propositions:

(74) $\emptyset$ is a set with measure zero w.r.t. B-Meas. The theorem is a consequence of $(72)$.

(75) Let us consider a real number $a$. Then $\{a\}$ is a set with measure zero w.r.t. B-Meas. The theorem is a consequence of (72).

(76) The Borel sets $\subseteq$ L-Field. The theorem is a consequence of (74).

(77) Let us consider an interval $A$. Then $($ L-Meas $)(A)=\varnothing A$. The theorem is a consequence of (73), (74), and (72).

\section{REFERENCES}

[1] Grzegorz Bancerek, Czesław Byliński, Adam Grabowski, Artur Korniłowicz, Roman Matuszewski, Adam Naumowicz, Karol Pąk, and Josef Urban. Mizar: State-of-the-art and beyond. In Manfred Kerber, Jacques Carette, Cezary Kaliszyk, Florian Rabe, and Volker Sorge, editors, Intelligent Computer Mathematics, volume 9150 of Lecture Notes in Computer Science, pages 261-279. Springer International Publishing, 2015. ISBN 978-3319-20614-1. doi:10.1007/978-3-319-20615-8_17.

[2] Grzegorz Bancerek, Czesław Byliński, Adam Grabowski, Artur Korniłowicz, Roman Matuszewski, Adam Naumowicz, and Karol Pakk. The role of the Mizar Mathematical Library for interactive proof development in Mizar. Journal of Automated Reasoning, 61(1):9-32, 2018. do1 10.1007 /s10817-017-9440-6

[3] Heinz Bauer. Measure and Integration Theory. Walter de Gruyter Inc., 2002.

[4] Józef Białas. The one-dimensional Lebesgue measure. Formalized Mathematics, 5(2):253258, 1996.

[5] Noboru Endou and Yasunari Shidama. Integral of measurable function. Formalized Mathematics, 14(2):53-70, 2006. doi 10.2478/v10037-006-0008-x.

[6] Noboru Endou, Hiroyuki Okazaki, and Yasunari Shidama. Hopf extension theorem of measure. Formalized Mathematics, 17(2):157-162, 2009. doi $10.2478 /$ v10037-009-0018-6.

[7] Gerald B. Folland. Real Analysis: Modern Techniques and Their Applications. Wiley, 2nd edition, 1999.

[8] Hiroshi Yamazaki, Noboru Endou, Yasunari Shidama, and Hiroyuki Okazaki. Inferior limit, superior limit and convergence of sequences of extended real numbers. Formalized Mathematics, 15(4):231-236, 2007. doi 10.2478/v10037-007-0026-3. 